\title{
AvaliaÇão do potencial de IMPaCto do liXiViado de aterro sanitário SOBRE ORGANISMOS AQUÁTICOS
}

\author{
Heider Alves Franco ${ }^{1 *}$, Sérgio Thode Filho ${ }^{2}$, Daniel Vidal Pérez ${ }^{3}$, Monica Regina da Costa Marques ${ }^{4}$
}

\author{
${ }^{1}$ Instituto Federal do Rio de Janeiro (IFRJ), Campus Pinheiral. Rua: José Breves, 550, Centro. Pinheiral/ RJ. \\ ${ }^{2}$ Instituto Federal do Rio de Janeiro (IFRJ), Campus Duque de Caxias. Av. República do Paraguai, 120 - Vila Sarapui, Duque de Caxias - RJ. \\ ${ }^{3}$ Empresa Brasileira de Pesquisa Agropecuária (EMBRAPA), Centro Nacional de Pesquisa em Solos. Rua Jardim Botanico, 1024, Jardim Botânico, Rio de \\ Janeiro, RJ. \\ ${ }^{4}$ Universidade do Estado do Rio de Janeiro (UERJ). Rua: São Francisco Xavier, 524, Maracanã, Rio de Janeiro - RJ. \\ * Autor para correspondência: heider.franco@ifrj.edu.br
}

Recebido em 20 de fevereiro de 2018. Aceito em 21 de dezembro de 2018. Publicado em 28 de dezembro de 2018.

REsumo - A geração de lixiviado de aterro sanitário ocorre devido à degradação dos resíduos sólidos que apresenta-se como um líquido potencialmente poluidor que pode acometer os recursos naturais nas proximidades. A avaliação desse risco pode ser realizada por testes de ecotoxicidade via determinação da concentração letal $\left(\mathrm{CL}_{50}\right)$. O objetivo deste estudo foi avaliar o impacto do lixiviado de aterro sanitário sobre organismos aquáticos, utilizando o Brachydanio rerio como organismo teste. Realizou-se um bioensaio do tipo estático, em aquário de vidro com volume total de 1,0 L; a água de preenchimento foi mantida sob temperatura em torno de $26 \pm 2{ }^{\circ} \mathrm{C}$, sem cloração, $\mathrm{pH} 7,0$ e OD $8 \mathrm{mg} \mathrm{L}^{-1}$ Cada aquário recebeu dez peixes com peso individual variando entre 80 e $100 \mathrm{mg}$, respeitando-se a densidade máxima de $1,0 \mathrm{~g} \mathrm{~L}{ }^{-1}$. Utilizou-se o delineamento inteiramente casualizado, composto de 6 tratamentos com níveis crescentes de lixiviado de aterro (1,0, 1,8, 2,0, 2,5, 2,7 e 2,8\%), diluídos em água deionizada, para o grupo teste e um grupo controle (água deionizada) ambos em triplicata. A CL de $2,35 \%$ de lixiviado reforça a necessidade do tratamento e/ou reuso como forma de minimizar os possíveis impactos ao meio, principalmente em organismos aquáticos.

Palavras-chave: Impacto Ambiental; Contaminação; Recursos Hídricos; Ecotoxicologia; Concentração Letal.

\section{ASSESSMENT OF THE IMPACT POTENTIAL OF LANDFILL LEACHATE ON AQUATIC ORGANISMS}

Aвstract - The generation of landfill leachate occurs due to the degradation of solid waste that presents itself as a potentially polluting liquid that can attack nearby natural resources. The assessment of this risk may be carried out by ecotoxicity tests by determining the lethal concentration $\left(\mathrm{LC}_{50}\right)$. The objective of this study was to evaluate the impact of landfill leachate in aquatic organisms, using Brachydanio rerio as a test organism. A static-type bioassay was performed in glass aquariums with a total volume of $1.0 \mathrm{~L}$, the filling water was maintained under temperature around $26 \pm 2{ }^{\circ} \mathrm{C}$, without chlorination, $\mathrm{pH} 7.0$ and OD $8 \mathrm{mgL}^{-1}$. Each aquarium received ten fish with individual weight that oscillated between 80 and $100 \mathrm{mg}$, respecting the maximum density of $1.0 \mathrm{~g} \mathrm{~L}^{-1}$. A completely randomized design was used, consisting of 6 treatments with increasing levels of landfill leachate $(1.0,1.8,2.0,2.5,2.7$ and 2.8\%), diluted in deionized water, for the test group and a control group (deionized water) both in triplicate. The $\mathrm{LC}_{50}$ of $2.35 \%$ of leachate reinforces the need for treatment and/or reuse as a means of minimizing possible impacts to the environment, especially on aquatic organisms.

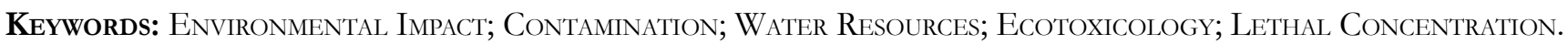




\section{EVALUACIÓN DEL POTENCIAL DE IMPACTO DEL LIXIVIADO DE VERTEDERO EN ORGANISMOS ACUÁTICOS}

Resumen - La generación de lixiviado de vertedero se produce debido a la degradación de los residuos sólidos que se presenta como un líquido potencialmente contaminante que puede acometer los recursos naturales cercanos. La evaluación de este riesgo puede realizarse a través de pruebas de ecotoxicidad mediante la determinación de la concentración letal (CL50). El objetivo de este estudio fue evaluar el impacto del lixiviado de vertedero en organismos acuáticos, utilizando el Brachydanio rerio como cuerpo de prueba. Se realizó un bioensayo del tipo estático, en acuario de vidrio con un volumen total de 1,0 L; el agua de relleno se mantuvo bajo temperatura alrededor de los $26 \pm 2{ }^{\circ} \mathrm{C}$, sin cloración, $\mathrm{pH} 7,0$ y OD $8 \mathrm{mg}$ L-1. Cada acuario recibió diez peces con peso individual que oscilaba entre 80 y $100 \mathrm{mg}$, respetando la densidad máxima de $1,0 \mathrm{~g} \mathrm{~L}-1$. Se utilizó el delineamiento completamente aleatorizado, compuesto de 6 tratamientos con niveles crecientes de lixiviado de vertedero $(1,0,1,8,2,0,2,5,2,7$ y $2,8 \%)$, diluidos en agua desionizada, para el grupo de prueba y un grupo control (agua desionizada) ambos en triplicado. La CL50 del 2,35\% de lixiviado refuerza la necesidad del tratamiento y / o reúso como forma de minimizar los posibles impactos al entorno, principalmente en organismos acuáticos.

Palabras clave: Impacto Ambiental; contaminación; Recursos hídricos; ecotoxicología; Concentración letal.

\section{INTRODUÇÃO}

A geração de lixiviado de aterro sanitário ocorre devido à degradação dos resíduos sólidos pela presença de microrganismos, em sua grande maioria bactérias atuantes no metabolismo aeróbio ou anaeróbio, caracterizados pela existência e pela ausência de oxigênio, respectivamente; além desses, as condições do meio, característica dos resíduos e precipitação contribuem para um maior ou menor volume de lixiviado (IPT 1995).

Especial atenção deve ser dada ao lixiviado, pois esse apresenta-se como um líquido potencialmente poluidor que pode acometer os recursos naturais nas proximidades, caso não seja tratado e descartado ao meio de maneira cuidadosa e controlada (Salem et al. 2008)

A caracterização do lixiviado de aterro identifica e quantifica as substâncias químicas e característica físicas do mesmo, contudo não é possível inferir seu impacto direto sobre o ecossistema. Para a análise dos efeitos tóxicos das substâncias ou misturas, descartadas/dispostas e/ou que venham a ser utilizadas nas atividades humanas, devem ser realizados testes de toxicidade ou bioensaios que visam predizer o impacto potencial de um xenobiótico (agente tóxico) ao ambiente (Flohr et al. 2005; Franco et al. 2017).

Os testes de ecotoxicidade ou bioensaios são realizados utilizando indivíduos, denominados como organismosteste. Esses, devido à sua característica de pequeno limite de tolerância ecológica, a determinadas substâncias químicas apresentam efeitos adversos, os quais são observados e quantificados, desde alterações genéticas, imobilidade, deformidades até letalidade (Marschner 1999; Lombardi 2004; Costa et al. 2008; Magalhães e Ferrão Filho 2008; Pimentel et al. 2011).

A intensidade dos efeitos depende da concentração, propriedades químicas das substâncias, sensibilidade do organismo teste e tempo de exposição. Desse modo, é recomendado que o efeito tóxico de uma substância seja avaliado com testes de toxicidade realizado em organismos de espécies e níveis tróficos diferentes (Costa et al. 2008).

O uso de bioensaios, em nível de organismo, é uma aplicação metodológica que se situa no meio da escala hierárquica de resposta a estressores, integrando os níveis bioquímico, celular e fisiológico. De tal forma, que testes a esse nível, fornecem uma boa avaliação do risco de extinção local de alguns organismos susceptíveis, antes que esses efeitos atinjam o nível de populações e comunidades (Magalhães e Ferrão Filho 2008).

Os diversos organismos teste prestam-se a uma gama de avaliações de impacto ambiental sobre o solo, as plantas e a água. Esses são selecionados em função da sua sensibilidade aos produtos ou compostos químicos. Desse modo, organismos aquáticos são utilizados para avaliação de impacto sobre corpos hídricos e/ou receptores (Schvartsman 1991). 
A ecotoxicologia aquática surgiu para dar suporte quanto aos problemas enfrentados pela contaminação dos corpos d'água por compostos tóxicos. Atua de maneira preditiva visando responder à toxicidade de compostos químicos, sinalizando os potenciais ecotoxicológicos e seus mecanismos de ação em organismos vivos, a partir da obtenção de curvas de concentração-efeito e concentração-reposta (Magalhães e Ferrão Filho 2008).

O risco de contaminação dos recursos hídricos, superficiais ou subterrâneos pelos lixiviados de aterro, dada sua complexa composição, é considerado um dos impactos mais significantes da deposição de resíduos em aterros sanitários (Kjeldsen e Christophersen 2001; Silva et al. 2004).

A avaliação desses riscos é caracterizada por diversos testes de ecotoxicidade, com a utilização de Vibrio fisheri, Daphnia similes, Artemia salina, Brachydanio rerio entre outros, como organismos teste (Sisinno et al. 2000; Renou et al. 2008).

Sendo assim, o presente trabalho objetivou avaliar o impacto do lixiviado de aterro sanitário sobre organismos aquáticos, utilizando o Brachydanio rerio como organismo teste.

\section{Material e Métodos}

A implantação e condução deste estudo ocorreu no Laboratório Multidisciplinar de Tecnologia Agroambiental do Campus Pinheiral do Instituto Federal do Rio de Janeiro, localizado no município de Pinheiral-RJ.

O planejamento, coleta, e conservação do lixiviado foram realizados de acordo com a NBR 9897 (ABNT 1987), bem como as análises físico-químicas para a caracterização do lixiviado de aterro e quanto à presença e concentração de metais pesados e sais (APHA 2005).

Para realização do bioensaio, foi utilizada uma espécie de peixe exótica ornamental, conhecida como paulistinha (Brachydanio rerio), comum em testes toxicológicos (Murty 1988; Patricio et al. 2002).

Esse procedimento é caracterizado como estático, pois não há substituição de água e nem retirada de detritos durante o período de exposição (48 horas) NBR 15088/2011 (ABNT 2011).

$\mathrm{O}$ acondicionamento dos peixes ocorreu em recipientes previamente preparados (aquário de vidro) com volume total de $1,0 \mathrm{~L}$, a água de preenchimento foi mantida sob temperatura em torno de $26 \pm 2{ }^{\circ} \mathrm{C}$, sem cloração, $\mathrm{pH}$ 7,0 e OD $8 \mathrm{mg} \mathrm{L}^{-1}$. Cada recipiente recebeu dez peixes com peso individual variando entre 80 e $100 \mathrm{mg}$, respeitando-se a densidade máxima de 1,0 $\mathrm{g} \mathrm{L}^{-1}$ (ABNT 2011).

Os ensaios foram realizados em caráter exploratório devido ao tipo de poluente (Aragão e Araújo 2008) utilizando-se doses decrescentes na razão de 50\%, que tiveram como partida a dosagem de $100 \%$ de lixiviado de aterro sanitário, ou seja, 100; 50; 25; 12,5; 6,25 e 3,125\%, diluídos em água deionizada. Entretanto, os resultados, causaram mortalidade instantânea dos organismos teste, fazendo-se necessária avaliação posterior com nova série de doses $0 \%$ (água deionizada), 1,8\%, 2,0\%, 2,5\%, 2,7\%, 2,8\%. Com isso, adotou-se o delineamento experimental inteiramente casualizado composto de 6 tratamentos para o grupo teste e um grupo controle. O grupo controle foi composto apenas de água deionizada. Para todos os tratamentos foram utilizadas 3 repetições.

As avaliações ocorreram pela contagem e retirada diária de animais mortos no período de 48 horas. O cálculo da $\mathrm{CL}_{50-48 h o r a s}$ foi realizado inicialmente determinando a regressão, descrevendo a função onde x refere-se aos tratamentos e y ao percentual de mortalidade de indivíduos. Na sequência, substitui-se o valor de y por 50 e, através da curva de dose-resposta, calculou-se a $\mathrm{CL}_{50-48 h o r a s}$ (ABNT 2011). A função foi determinada com 95\% de confiabilidade.

Adicionalmente, procedeu-se a análise da significância das concentrações entre as triplicatas pelo teste de SCOTT-KNOTT (1974) à 5\% de probabilidade.

As análises foram realizadas com auxílio do programa Assistat 7.7 (Silva e Azevedo 2016) e os figuras construídos utilizando o programa QtiPlot. 


\section{Resultados e Discussão}

De acordo com os parâmetros físico-químicos (Tabela 1) observa-se que os elementos amônio, cloreto, lítio, nitrato, sulfato além da DQO, estão acima dos valores de referência (CONAMA 430). Desse modo, fica impedido o lançamento do efluente em corpo receptor. O mesmo, foi observado por Franco et al. (2017).

Tabela 1 - Caracterização física e química do lixiviado de aterro sanitário do CTR de Barra Mansa/RJ

\begin{tabular}{|c|c|c|c|c|}
\hline \multirow{2}{*}{ Parâmetros avaliados } & \multirow{2}{*}{ Unidade } & \multirow{2}{*}{ Valores observados } & Resolução $n^{\circ} 430^{1}$ & Resolução $n^{\circ} 430^{2}$ \\
\hline & & & \multicolumn{2}{|c|}{ (CONAMA, 2011) } \\
\hline pH (água) & $\begin{array}{c}------- \\
-1\end{array}$ & 9,0 & $5,0-9,0$ & $6,0-9,0$ \\
\hline Condutividade elétrica & $\mathrm{dS} \mathrm{m}^{-1}$ & 33,3 & N.D. & N.D. \\
\hline DQO & $\mathrm{mg} \mathrm{L}^{-1}$ & 5592 & 120 & N.D. \\
\hline Sólidos totais dissolvidos & $\mathrm{mg} \mathrm{L}^{-1}$ & 23600 & N.D. & 500 \\
\hline Salinidade & $\mathrm{mg} \mathrm{L} \mathrm{L}^{-1}$ & 16900 & N.D. & N.D. \\
\hline Amônio** & $\mathrm{mg} \mathrm{L}^{-1}$ & 595,27 & 20,0 & N.D. \\
\hline Cádmio* & $\mathrm{mg} \mathrm{L} \mathrm{L}^{-1}$ & 0,025 & 0,2 & 0,01 \\
\hline Cálcio** & $\mathrm{mg} \mathrm{L}^{-1}$ & 95,38 & N.D. & N.D. \\
\hline Chumbo* & $\mathrm{mg} \mathrm{L}^{-1}$ & 0,37 & 0,5 & 0,033 \\
\hline Cloreto** & $\mathrm{mg} \mathrm{L}^{-1}$ & 857,50 & 250,0 & 250,0 \\
\hline Cobre* & $\mathrm{mg} \mathrm{L}^{-1}$ & 0,17 & 1,0 & 0,013 \\
\hline Ferro* & $\mathrm{mg} \mathrm{L}^{-1}$ & 13,66 & 15,0 & 5,0 \\
\hline Lítio** & $\mathrm{mg} \mathrm{L}^{-1}$ & 15,22 & 2,5 & 2,5 \\
\hline Magnésio** & $\mathrm{mg} \mathrm{L}^{-1}$ & 15,37 & N.D. & N.D. \\
\hline Niquel* & $\mathrm{mg} \mathrm{L}^{-1}$ & 0,48 & 2,0 & 0,025 \\
\hline Nitrato** & $\mathrm{mg} \mathrm{L}^{-1}$ & 496,75 & 10,0 & 10,0 \\
\hline Potássio** & $\mathrm{mg} \mathrm{L}^{-1}$ & 694,46 & N.D. & N.D. \\
\hline Sódio** & $\mathrm{mg} \mathrm{L}^{-1}$ & 1172,46 & N.D. & N.D. \\
\hline Sulfato** & $\mathrm{mg} \mathrm{L}^{-1}$ & 846,00 & 250,0 & 250,0 \\
\hline Zinco* & $\mathrm{mg} \mathrm{L}^{-1}$ & 1,13 & 5,0 & 5,0 \\
\hline
\end{tabular}

pH - potencial hidrogeniônico; DQO - Demanda Química de Oxigênio; *Leitura via absorção atômica; ** Leitura via cromatógrafo de íons; N.D. não detectado; ${ }^{1}$ Padrão para lançamento de efluentes em corpos receptores; ${ }^{2}$ Padrão para água doce classe 3.

Fonte: Franco et al. (2017)

Verifica-se também que quando se compara os valores observados no lixiviado de aterro sanitário bruto (in natura) com o padrão para água doce classe 3, o mesmo não possui característica para ser utilizado como água para: a) abastecimento e consumo humano, após tratamento convencional ou avançado; b) irrigação de culturas arbóreas, cerealíferas e forrageiras; c) pesca amadora; d) recreação de contato secundário (CONAMA 430).

Os valores médios do percentual de mortalidade em função dos diferentes tratamentos com lixiviado estão apresentados na figura 1. Observou-se uma relação quadrática entre o percentual de mortalidade e o aumento da dose do lixiviado. Não foram observadas diferenças significativas entre os tratamentos 1,8 e 2,0\% de lixiviado os quais apresentaram mortalidade média de $15 \%$ e, entre os 2,5, 2,7 e 2,8\% cuja mortalidade média foi de $95 \%$. 
Figura 1. Mortalidade de Brachydanio rerio em função de doses de lixiviado de aterro sanitário.



Legenda: Médias seguidas pela mesma letra minúscula não diferem estatisticamente entre si pelo teste de SCOTT-KNOTT a $5 \%$ de probabilidade.

A CL $\mathrm{L0}_{50-48 h o r a s}$ do bioensaio foi calculada a partir da função quadrática descrita por $\mathrm{y}=54,1481 \mathrm{x}^{2}-144,4436 \mathrm{x}+$ 90,2517 com $\mathrm{R}^{2}=0,9356$ (figura 1). Utilizando essa função e substituindo y por 50, encontrou-se o valor de 2,35\% como sendo a dose de lixiviado $\left(\mathrm{CL}_{50-48 \mathrm{hora}}\right)$ capaz de causar letalidade em $50 \%$ dos organismos teste.

Silva et al. (2003) avaliaram a sensibilidade de Brachydanio rerio e Poecilia vivipara (Pisces) ao chorume bruto do aterro de Gramacho/RJ, identificando a dose de $2,24 \%$ como a $\mathrm{CL}_{50}$ para Danio rerio, corroborando assim com o presente estudo.

Ainda quanto à toxicidade de lixiviado de aterro sobre organismos aquáticos, Daflon et al. (2012) determinaram a $\mathrm{CL}_{50}$ para Danio rerio com dose de $0,45 \%$ e mesmo após tratamento por clarificação consideraram o poluente com grande toxicidade dada a $\mathrm{CL}_{50}$ igual a $4,47 \%$ para o mesmo organismo teste.

Outros autores ao estudarem o efeito agudo de defensivos agrícolas como o Parathion metil sobre Danio rerio, encontraram valores para $\mathrm{CL}_{50}$ de 1,9 mg/L (Roex et al. 2002), 5,6 mg/L (Bellavere e Gorbi 1984) e 14,1 $\mathrm{mg} / \mathrm{L}$ (Quaresma et al. 2008).

O ensaio de efeito agudo é definido como sendo uma resposta severa e rápida dos organismos aquáticos a um estímulo que pode se manifestar num curto período de até 48 horas, causando quase sempre a letalidade (CETESB 1990). Normalmente, estes efeitos são observados quando efluentes são despejados ao corpo hídrico sem passar por tratamento, causando letalidade a organismos pertencentes a diferentes níveis tróficos (Bassoi et al. 1990).

Estes testes são aplicados também para avaliar a sensibilidade relativa de organismos aquáticos para um determinado agente tóxico, para determinar a qualidade de água e as concentrações seguras de agentes químicos para preservação da vida aquática, além de avaliar a fertilidade potencial das águas e a eficiência de diferentes métodos de tratamento para efluentes industriais em termos toxicológicos (Metcalf e Eddy 2003).

\section{Conclusão}

A partir dos ensaios realizados, a $\mathrm{CL}_{50-48 h o r a s}$ foi obtida na dose com 2,35\% de lixiviado reforçando a necessidade do tratamento e/ou reuso como forma de minimizar os possíveis impactos ao meio, principalmente em organismos 
aquáticos. Ainda nesse contexto, pode-se avaliar que o resultado aqui encontrado, segue de acordo com aqueles apresentados na literatura avaliando o efeito de diferentes substâncias sobre Brachydanio rerio.

Reforça-se ainda a importância da ecotoxicologia, através dos ensaios de letalidade como ferramenta para determinação do impacto ambiental de efluentes potencialmente contaminantes. Os ensaios ecotoxicológicos utilizando Bracbydanio rerio, como organismo teste, bioindicador, apresentam-se como ferramenta para a avaliação do potencial de impacto ambiental do lixiviado de aterro sanitário sobre corpos hídricos receptores. Corroborando ainda com uma abordagem multidisciplinar que implica na necessidade de realizar diferentes avaliações para a determinação do potencial de impacto ambiental de uma substância, principalmente em recursos hídricos, orientando sobre a necessidade de tratamento para posterior disposição.

\section{Agradecimentos}

À FOXX HAZTEC pelo fornecimento do lixiviado de aterro sanitário e ao IFRJ-Campus Pinheiral, pelo apoio estrutural à pesquisa.

\section{REFERÊNCIAS}

APHA. AMERICAN PUBLIC HEALTH ASSOCIATION. 2005. Standard methods for examination of water and wastewater. Washington: American Water Works Association, 21th ed. 1.368p.

Aragão MA, Araújo RPA. 2008. Métodos de ensaios de toxicidade com organismos aquáticos. In ZAGATTO, P.A.; BERTOLETTI, E. (Editores). Ecotoxicologia Aquática: Princípios e Aplicações, São Carlos, São Paulo: RIMA, p. 117 $-147$.

\section{ASSOCIAÇÃO BRASILEIRA DE NORMAS TÉCNICAS - ABNT.}

. NBR 8412: Apresentaşão de projetos de aterros sanitários de resíduos sólidos urbanos - Procedimento. NBR-8419/92. São Paulo, 13p. 1992.

NBR 15088: Ecotoxicologia aquática - Toxicidade aguda - Método de ensaio com peixes - Procedimento. NBR 15088/2011. São Paulo, 22p. 2011.

NBR 9897: Planejamento de amostragem de efluentes líquidos e corpos receptores - Procedimento. NBR - 9897/87. Rio de Janeiro, 22p. 1987.

Bassoi LJ, Nieto R, Tremaroli D. 1990. Implementação de testes de toxicidade no controle de efluentes líquidos. São Paulo: CETESB, p. 7.

Bellavere C, Gorbi G. 1984. Biological variability and acute toxicity of Parathion, Dichlobenil and TPBS to Biomphalaria glabrata and Brachydanio rerio. Environ. Technol. Lett., v. 5, n. 9, p. 389-396.

COMPANHIA DE TECNOLOGIA DE SANEAMENTO AMBIENTAL - CETESB. Procedimentos para utilização de testes de toxicidade no controle de efluentes líquidos. São Paulo: CETESB, 1990. p. 17.

CONAMA - Conselho Nacional do Meio Ambiente. Resolução n: 430/2011, de 13/05/2011. Disponível em: http://www. mma.gov.br/port/conama/legiabre.cfm?codlegi=646. Acesso em 10 Jul. 2017. 
Costa CR, Olivi P, Botta CM, Espindola ELG. 2008. A toxicidade em ambientes aquáticos: discussão e métodos de avaliação. Quim Nova. 31 : 1820-1830.

Costa Neto PLO. Estatística. São Paulo: Edgard Blücher, 1977. 264p.

Daflon SDA, Giordano G, Filho OB. 2012. Avaliação da redução da toxicidade no tratamento do chorume de São Paulo com dois organismos-teste. XII Congresso Brasileiro de Ecotoxicologia. 25 a 28 de Setembro de 2012, Porto de Galinhas PE.

Flohr L, Brentano DM, Carvalho-Pinto CRS, Machado VG, Matias WG. 2005. Classificação de resíduos sólidos industriais com base em testes ecotoxicológicos utilizando Daphnia magna: Uma alternativa. Biotemas, 18:7-18.

Franco HA, Marques MR da C, Martins GM de, Mussel YL, Thode Filho S. Ecotoxicological evaluation of the application of landfill leachate on the germination of cabbage (Brassica oleracea var. capitata). Revista Eletrônica em Gestão, Educação e Tecnologia Ambiental. v.21, SPECIAL EDITION, 2017, p. 45-57.

Hamilton MA, Russo RC, Thurston RV. 1977. Trimmed Spearman-Karber method for estimating median lethal concentrations in toxicity biossays. Environmental Science and Technology, 11(7): 714-719.

INSTITUTO DE PESQUISAS TECNOLÓGICAS DO ESTADO DE SÃO PAULO (IPT). 1995. Lixo Municipal: manual de gerenciamento integrado. São Paulo: IPT/CEMPRE. 278p.

Kjeldsen P, Christophersen M. 2001. Composition of leachate from old landfills in Denmark. Waste Management \& Research, v. 19, n. 3, p. 249-256.

Lombardi JV. 2004. Fundamentos de toxicologia aquática. Pp. 261 - 270 In: M. J. T. Ranzani - Paiva, R. M. Takemota \& M. A. P. Lizama (Eds.), Sanidade de organismos aquáticos. Org. Livraria Varela, São Paulo, 426p.

Magalhães DP, Ferrão Filho AS. 2008. A ecotoxicologia como ferramenta no biomonitoramento de ecossistemas aquáticos. Oecol. Bras., 12 (3): 355 - 381.

Marschner A. 1999. Biologische Bodensanierung und ihre Erfolgskontrolle durch Biomonitoring. Pp. 568 - 576. In: J. Oehlmann \& B. Markert (Eds.), Õkotoxikologie - Õkosystemare Ansãtze und Methoden. Ecomed, Landsberg, 576p.

Metcalf L, Eddy HP. 2003. Wastewater engineering treatment in reuse. 4.ed. Mcgraw Hill: Boston.

Murty AS. 1998. Toxicity of pesticide to fish. USA, CRC press, (1), 192p.

Patricio FC, Rigitano RLO, Gouvêa AV, Franco AA. 2002. Toxicidade do inseticida-nematicida Aldicarbe às espécies de peixes Brachydanio rerio e Orthospinusfranciscensis. Ciência e Agrotecnologia, Lavras, (26) 2, 385-391.

Pimentel MF, Silva Júnior FCG, Santaella ST, Lotufo LVC. 2011. O uso de Artemia sp. como organismo-teste para avaliação da toxicidade das águas residuárias do beneficiamento da castanha de caju antes e após tratamento em reator biológico experimental. Journal of the Brazilian Society of Ecotoxicology, v. 6, n. 1, p. 15-22.

QtiPlot: data analysis and scientific visualization. 2018. Disponível: http://soft.proindependent.com/qtiplot.html. Acesso em: 16 Fev. 
Quaresma CC, Garcia MVB, Garcia TB. 2008. Toxicidade Aguda de Parathion Metil para Danio rerio (Teleostei, Cyprinidae), em condições Tropicais. Anais da III Jornada de Iniciação Científica da Embrapa Amazônia Ocidental.

Renou S, Givaudan JG, Poulain S, Dirassouyan F, Moulin P. 2008. Landfill leachate treatment: Review and opportunity. Journal of Hazardous Materials, 150, 468-493.

Roex EWM, Van Langen MCT, Van Gestel CAM. 2002. Acute toxicity of two compounds with different modes of action to the zebrafish, Danio rerio. Bull. Environ. Contam. Toxicol., v. 68, p. 269- 274.

Salem Y, Hamouri K, Djemaa R, Alois K. 2008. Evaluation of landfill leachate pollution and treatment. Desalination, 220, $108-114$.

Schvartsman S. 1991. Intoxicações agudas. 4ed. Ed. Sarvier, São Paulo, 355p.

Scott AJ, Knott M. 1974. "A cluster analysis method for grouping means in the analysis of variance." Biometrics, 507-512.

Silva AC, Bonecker ACT, Dezotti M, Sant'anna JR, GL. 2003. Avaliação da Sensibilidade de Bracbydanio rerio e Poecilia vivipara (Pisces) ao chorume do aterro de Gramacho/RJ. 22 ${ }^{\circ}$ Congresso Brasileiro de Engenharia Sanitária e Ambiental. 14 a 19 de Setembro de 2003, Joinville - Santa Catarina.

Silva AC, Dezotti M, Sant'anna JR. GL. 2004. Treatment and detoxification of a sanitary landfill leachate.Chemosphere, v. 55, n. 2, p. 207-214.

Silva F de AS, Azevedo CAV de. 2016. The Assistat Software Version 7.7 and its use in the analysis of experimental data. Afr. J. Agric. Res, v.11, n.39, p.3733-3740. DOI: 10.5897/AJAR2016.11522.

Sisinno CLS, Oliveira-Filho EC, Dufrayer EMC. 2000 Toxicity evaluation of a municipal dump leachate using zebrafish acute tests. Bulletin of Environmental Contamination and Toxicology 64: 107-113.

USEPA - UNITED STATES ENVIRONMENTAL PROTECTION AGENCY. 1998. SW-846 EPA Method 3051A. Microwave assisted acid digestion of sediments, sludges, soils and oils. Test Methods for Evaluating Solid Waste. 3rd Update. Washington, DC. 